\title{
Guest Editor: Benjamin Bonavida
}

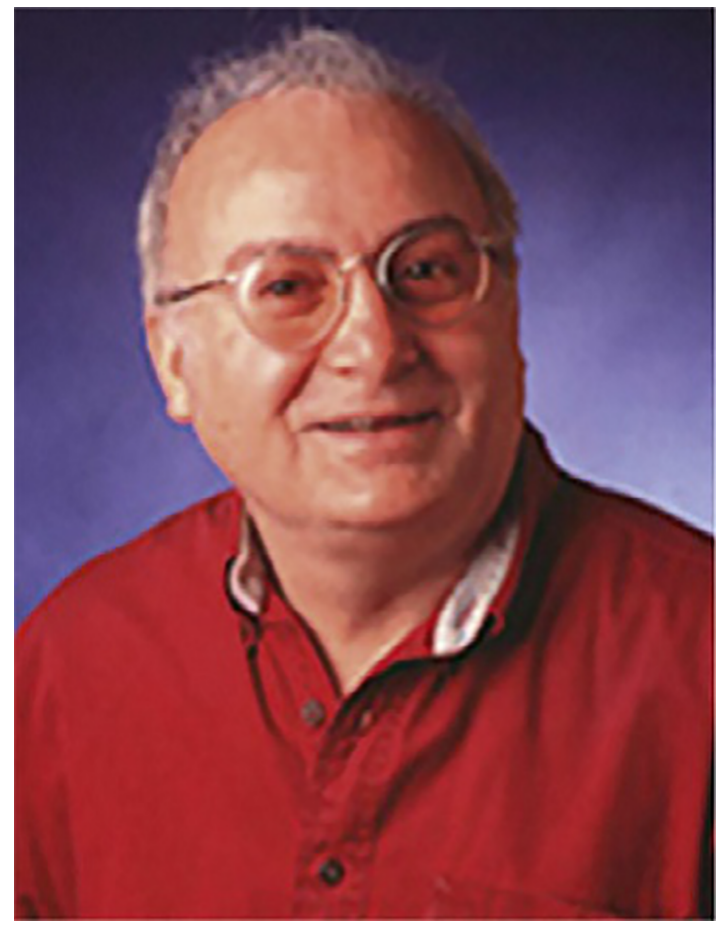

Dr. Benjamin Bonavida, PhD, Editor-in-Chief, is currently Distinguished Research Professor at the University of California, Los Angeles (UCLA). He is affiliated with the Department of Microbiology, Immunology and Molecular Genetics, UCLA David Geffen School of Medicine. His research career, thus far, has focused on investigations in the fields of basic immunochemistry and cancer immunobiology. His research investigations have ranged from the biochemical, molecular, and genetic mechanisms of cell-mediated killing and tumor cell resistance to chemo-/immunocytotoxic drugs. The reversal of tumor cell resistance was investigated by the use of various selected sensitizing agents based on molecular mechanisms of resistance. In these investigations, there was the newly characterized dysregulated NF- $\kappa \mathrm{B} /$ Snail/YY1/RKIP/PTEN loop in many cancers that was reported to regulate cell survival, proliferation, invasion, metastasis, and resistance. Emphasis was focused on the roles of the tumor suppressor Raf kinase inhibitor protein (RKIP), the tumor promoter Yin Yang 1 (YY1) and the role of nitric oxide as a chemo-/immunosensitizing factor. Many of the above studies are centered on the clinically challenging features of cancer patients' failure to respond to both conventional and targeted therapies.

Dr. Bonavida has been active in the organization of regular sequential international mini conferences that are highly focused on the roles of YY1, RKIP, and nitric oxide in cancer and their potential therapeutic applications. He has edited and coedited several books, and has been the Series Editor of more than 25 books published by Springer on Resistance to Anti-Cancer Targeted Therapeutics. He is presently the Series Editor of three series published by Elsevier/Academic Press on Chemotherapy-Sensitizing Agents for Cancer, Sensitizing Agents for Cancer Resistance to Antibodies, and Breaking Tolerance to Anti-Cancer Immunotherapy. He is the Editor-in-Chief of the journal Critical Reviews ${ }^{\mathrm{TM}}$ in Oncogenesis and Associate Editor of Critical Reviews ${ }^{\mathrm{TM}}$ in Immunology. He has published over 500 research publications and reviews in various scientific journals of high impact.

\section{ACKNOWLEDGMENTS}

The Guest Editor, Dr. Bonavida, acknowledges the Department of Microbiology, Immunology and Molecular Genetics and the UCLA David Geffen School of Medicine for their continuous support. The Guest Editor also acknowledges the excellent editorial assistance of Ms. Inesa Navasardyan. 